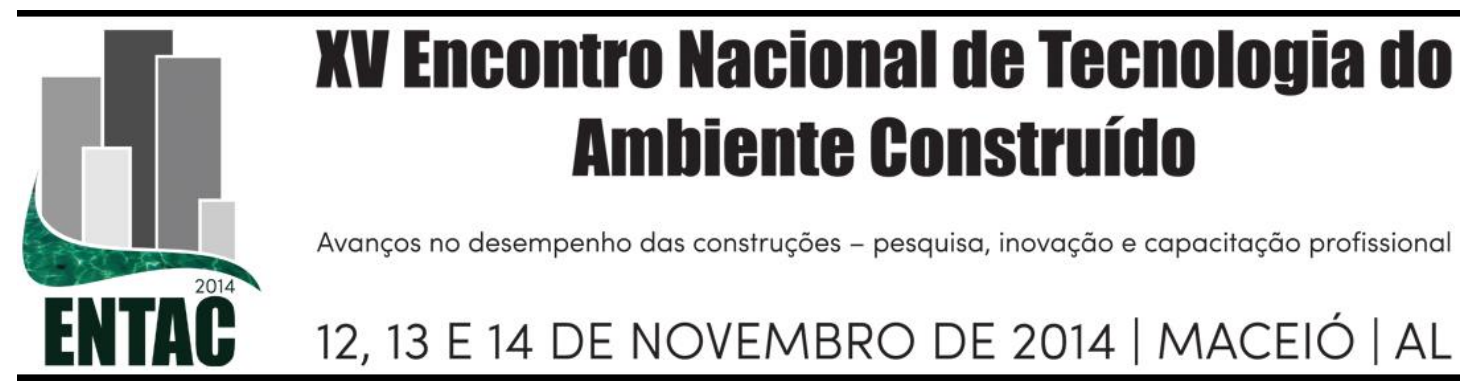

\title{
O USO DE MATERIAIS COM RESÍDUOS POLIMÉRICOS NO ISOLAMENTO ACÚSTICO DE FECHAMENTOS COMPOSTOS
}

\author{
BAYER, Elaine Cristina (1); DE OLIVEIRA NUNES, Maria Fernanda (2); \\ DE MORAES DE ZORZI, Lizia (3)
}

(1) Universidade de Caxias do Sul, e-mail: elainebayer@ hotmail.com (2) Universidade de Caxias do Sul, e-mail: mfonunes@ucs.br (3) Universidade Federal do Rio Grande do Sul, e-mail: liziadz@gmail.com

\begin{abstract}
RESUMO
Este estudo, de caráter exploratório, se justifica pela busca por maior conforto ambiental e acústico, impulsionados pela crescente exigibilidade das normas de desempenho, e pela busca por alternativas de materiais mais sustentáveis, produzidos a partir de resíduos. Ele faz parte da pesquisa APOTA, de Avaliação do Potencial Termo-Acústico de novos materiais com resíduos industriais, desenvolvida na Universidade de Caxias do Sul e tem por objetivo avaliar o desempenho de materiais confeccionados com resíduos poliméricos de sapatos (PU e EVA) no isolamento ao ruído aéreo em fechamentos verticais compostos, para que estes possam servir como alternativas com menor impacto ambiental e valor. Para a análise foram realizados ensaios in loco fundamentados na ISSO 140-5 e na ISSO 717-1 e testados os seguintes materiais: PU resíduo 15\%, três PUs juntos com resíduos de 0\%, 5\% e 10\%, EVA resíduo 40\% com amostras de 20x18 cm, EVA resíduo 40\% com amostras de 90x70 cm, e lã de vidro. Observou-se que todas as configurações apresentaram comportamento semelhante de 100 a $200 \mathrm{~Hz}$ possuindo nível sonoro inferior ao painel simples e de 1600 a $3150 \mathrm{~Hz}$ apresentando níveis aproximados ao do fechamento simples. A lã de vidro obteve o melhor resultado, seguida pela divisória cujo recheio foi constituído por três PUs resíduo $(0 \%+5 \%+10 \%)$, que apresentou comportamento muito semelhante ao primeiro a partir de $630 \mathrm{~Hz}$. Observou-se também que o EVA resíduo $40 \%$ com placa de $90 \times 70 \mathrm{~cm}$ apresentou o pior desempenho entre os materiais isolantes ensaiados. Os melhores desempenhos foram respectivamente: lã de vidro, três PUs resíduo, PU resíduo 15\% e EVA resíduo 40\% (20x18 cm). O EVA resíduo 40\% (90x70 cm) não apresentou melhora no isolamento do fechamento.
\end{abstract}

Palavras-chave: Isolamento acústico, Fechamentos compostos, Reuso de resíduos poliméricos.

\begin{abstract}
This study is justified by the search for greater environmental and acoustic comfort, driven by increasing enforceability of performance standards, and the search for alternative more sustainable materials produced from waste. It is part of APOTA, Evaluation Research Potential Thermo-Acoustic new materials with waste from footwear industry, developed at the University of Caxias do Sul and aims to evaluate the performance of materials made from polymeric waste of shoes (PU and EVA) airborne sound insulation in composite vertical locks, so that they can serve as alternatives with less environmental impact and value. For the analysis in situ tests based on ISO 140-5 and ISO 717-1 testing the following materials were performed: $15 \%$ PU residue three PUs together with waste 0\%, 5\% and $10 \%, 40 \%$ EVA residue with samples of $20 \times 18 \mathrm{~cm}, 40 \%$ EVA residue on samples of $90 \times 70 \mathrm{~cm}$, and glass wool. It was observed that all configurations have similar behavior 100-200 Hz having less than the single and 1600-3150 $\mathrm{Hz}$ presenting approximate the simple closing levels sound level. Glass wool obtained the best results, followed by the partition whose filling was made up of three PUs residue $10 \%+$ $5 \%+10 \%$ ), which showed very similar behavior to the first from $630 \mathrm{~Hz}$ was also observed that the EVA residue with $40 \% 90 \times 70 \mathrm{~cm}$ plate showed the worst performance among the tested insulating materials. The best performances were: glass wool, PUs three residue, residue 15\% PU and 40\% EVA residue $(20 \times 18 \mathrm{~cm})$. The residue EVA $40 \%(90 \times 70 \mathrm{~cm})$ showed no improvement in the isolation of closing.
\end{abstract}




\section{INTRODUÇÃO}

A importante concentração da população em centros urbanos gera uma demanda cada vez maior por edificações multifamiliares, visando otimizar a ocupação dos lotes urbanos. Nesse tipo de edificação com frequência podem ser verificados transtornos causados pelo excesso de ruído entre apartamentos e até ambientes. Junto com a crescente produtividade na construção civil, aumentam as exigências com relação ao desempenho das edificações, principalmente residenciais. No Brasil, no ano de 2013 entrou em vigor a Norma NBR 15575, que trata do desempenho dos principais elementos que compõem as edificações residenciais. Com a Norma são estabelecidos níveis de isolamento ao ruído, aumentando a exigência por isolamento ao ruído aéreo.

Além disso, desde a década de 1990 há uma crescente preocupação com o meio ambiente, com o consumo dos recursos naturais e com a sustentabilidade. De acordo com Dixit et al (2010) as edificações consomem aproximadamente 37\% da energia total global na forma de energia incorporada durante as fases de construção e de operação. Sendo a construção civil uma das grandes responsáveis por este consumo, é importante a busca por materiais alternativos e reciclados. Outro problema que o consumo exagerado provoca é a grande quantidade de resíduos que gera. A reciclagem de materiais pode ser vista, desta forma, como uma maneira de reduzir o consumo dos recursos naturais, evitar a disposição deles em lixões a céu aberto, como frequentemente acontece, e reduzir os impactos ambientais.

Estas crescentes preocupações com o desempenho e com a sustentabilidade justificam este estudo de caráter exploratório. Desenvolvido dentro da pesquisa APOTA, de Avaliação do Potencial Termo-Acústico de novos materiais com resíduos industriais, da Universidade de Caxias do Sul, este trabalho tem como objetivo avaliar o desempenho de materiais confeccionados com resíduos poliméricos da indústria calçadista no isolamento ao ruído aéreo em fechamentos verticais compostos para que estes possam ser alternativas de produtos com menor impacto ambiental e menor valor do que os derivados do petróleo utilizados atualmente.

Os materiais aqui estudados foram desenvolvidos a partir de resíduos de copolímero de etileno-acetato de vinila (EVA) e de poliuretano (PU) da indústria calçadista. Para a avaliação foram realizados ensaios in loco de acordo com as normas ISSO 140-5 e na ISSO 717-1, que tratam deste tipo de medição, e testados os seguintes materiais: PU resíduo $15 \%$, três PUs juntos com resíduos de $0 \%, 5 \%$ e 10\%, EVA resíduo $40 \%$ com amostras de 20x18 cm, EVA resíduo 40\% com amostras de 90x70 cm, e lã de vidro. Por fim, foi comparado atendimento às exigências de desempenho destes materiais com o da lã de vidro, material convencional mais utilizado para isolamento aos ruídos aéreos.

\section{FUNDAMENTAÇÃO}

De acordo a NBR 12179 (ABNT, 1992), som é "toda e qualquer vibração ou onda mecânica que se propaga num meio dotado de forças internas, capaz de produzir no homem uma sensação auditiva". Já o ruído é caracterizado como uma "mistura de sons cujas frequências não seguem nenhuma lei precisa, e que diferem entre si por valores imperceptíveis ao ouvido humano", e como todo o som indesejável (ANBT, 1983). O ruído pode ser classificado como de impacto ou aéreo. O ruído de impacto, também conhecido como estrutural, é transmitido pela vibração de um corpo sólido, como a estrutura de uma edificação e as paredes, apresentando picos de energia acústica de duração menor do que um segundo e com intervalos maiores do que um segundo como, 
por exemplo, passos. Já o ruído aéreo é o som ou ruído transmitido através do ar, como vozes e buzinas, por exemplo (VALLE, 2006; CARVALHO, 2006).

O isolamento acústico é a capacidade de impedir que um som ou ruído entre ou saia de um recinto fechado ou semi-fechado, abrangendo tanto o ruído aéreo, quanto o ruído de impacto (VALLE, 2006). Quando uma frente de onda encontra um fechamento vertical sólido, essa onda percorre toda a espessura do fechamento. A isolação sonora de uma parede é determinada por uma grandeza derivada do coeficiente de transmissão sonora (t) denominada perda de transmissão sonora (PT) (BISTAFA, 2006). Quanto menor for o coeficiente de transmissão, mais isolante será a parede, e, consequentemente, maior será a perda de transmissão.

Painéis homogêneos possuem uma curva típica de perda de transmissão em relação à frequência do som incidente. De acordo com Bistafa (2006), essa curva apresenta comportamentos distintos, sendo, por isso, dividida em quadro partes: a região controlada pela rigidez e ressonância, nas baixas frequências; a controlada pela massa, nas médias frequências e a região controlada pela coincidência, nas altas frequências. $\mathrm{Na}$ região controlada pela rigidez, a perda de transmissão diminui com variação linear conforme as frequências vão aumentando. Já na região controlada pela massa ocorre o inverso, a perda de transmissão aumenta com variação linear conforme o aumento das frequências. No entanto, as regiões controladas pela ressonância e coincidência, apresentam variação de acordo com nível de amortecimento do painel, não demonstrando variação linear.

Nessas regiões existem picos de redução da perda de transmissão, denominados frequência de ressonância (fr) e frequência crítica (fc). A frequência de ressonância ocorre quando as ondas de flexão geram combinações construtivas que se propagam pelo painel e são refletidas pelas suas bordas. Para compreender a frequência crítica é necessário conhecer a frequência de coincidência, que consiste basicamente na igualdade do comprimento de onda no ar com o comprimento de onda de reflexão no painel. Nas frequências de coincidência, o som incidente é transmitido por ele com eficiência, gerando a diminuição da perda de transmissão. A menor frequência de coincidência é denominada frequência crítica (fc), e ocorre quando a onda se propaga a rasante ao painel, ou seja, quando $\theta=90^{\circ}$ (BISTAFA, 2006).

Para que o isolamento acústico de um fechamento vertical seja eficiente, alguns cuidados são importantes. Bistafa (2006) recomenda o uso de paredes duplas quando quer se obter grande perda na transmissão, menor custo, ou peso. Valle (2006) complementa que as chapas da parede dupla devem ser isoladas entre si. Quanto maior o espaço entre as placas e a presença de material absorvente, maior é a perda de transmissão, assim como, quanto mais espesso for o material absorvente, maior será a atenuação do ruído. Esse princípio é denominado massa-mola-massa, sendo que a massa são as placas e a mola é o material absorvente (BISTAFA 2006). Com este tipo de fechamento composto, sé possível obter um bom isolamento acústico, pois o ruído tem que passar por mais barreiras com diferentes características.

\section{MATERIAIS E MÉTODOS}

Nesta pesquisa foi avaliado o isolamento acústico em fechamentos compostos com materiais produzidos a partir de resíduos industriais de copolímero de etileno-acetato de vinila (EVA) e de poliuretano (PU). Para que fosse possível a comparação do desempenho dos materiais, também foram realizados ensaios com lã de vidro, material convencional utilizado para isolamento acústico em fechamentos compostos. 
O EVA é um copolímero termofixo, podendo ser fundido apenas uma única vez. Em função disso, a indústria calçadista gera grande quantidade de retalhos do material no processo de fabricação de solas, entressolas e palmilhas. As amostras de EVA que foram analisadas nesta pesquisa contêm $40 \%$ de resíduo e possuem dois tamanhos distintos: um de 20 x $18 \mathrm{~cm}$ com $6 \mathrm{~mm}$ de espessura e outro de $90 \times 70 \mathrm{~cm}$ com $10 \mathrm{~mm}$ de espessura, confeccionadas por Menegotto (2009) e Rauber (2010), respectivamente. Em ambos os casos as amostras foram unidas formando placas com aproximadamente $1,1 \mathrm{~m}^{2}$ de área.

O PU é um polímero termofixo de baixa densidade $\left(16-48 \mathrm{~kg} \mathrm{~m}^{3}\right)$ com células fechadas, o que contribui também para a rigidez da espuma, apresentando uma estrutura altamente reticulada. Esse material é bastante utilizado, na indústria calçadista, na fabricação de solados. Por possuir características plásticas, com alta incidência na forma de espuma, acredita-se que seu resíduo possua grande potencial como material isolante sonoro (BECKER, 2002). As amostras de PU que foram analisadas nesta pesquisa são com $0 \%, 5 \%, 10 \%$ e $15 \%$ de resíduo e possuem dois tamanhos distintos. As amostras de PU com 0\%, 5\% e 10\% de resíduo, com $25 \times 25 \mathrm{~cm}$ e espessura de 10 $\mathrm{mm}$, foram confeccionadas por Silva (2010). E, as amostras de PU 15\%, com de 18 x 13 $\mathrm{cm}$ e espessura de $5 \mathrm{~mm}$, foram confeccionadas por Menegotto (2009). Neste estudo foram ensaiadas duas configurações com estas amostras: uma com o PU 15\% confeccionado por Menegotto, e outra com os três PUs confeccionados por Silva juntos $(0 \%, 5 \%$ e $10 \%)$. As amostras foram unidas de acordo com o seu percentual de resíduo e cada tipo totalizou aproximadamente $1,1 \mathrm{~m}^{2}$ de área.

Os ensaios foram realizados em um dormitório do alojamento da Universidade de Caxias do Sul. Esse ambiente, com volume de $22,7 \mathrm{~m}^{3}$, foi utilizado como câmara de recepção. A sua parede externa é bastante espessa (possuindo entre 22,5 e $36 \mathrm{~cm}$ ), sendo adequada para a realização do estudo devido a sua grande massa e, consequente, elevado isolamento acústico. A fonte sonora foi posicionada em ambiente externo, sob um terraço, para que houvesse uma melhor propagação do som emitido. Para a realização do ensaio foi utilizado o vão da janela existente no dormitório, como pode ser observado na Figura 1. A abertura é em madeira, com uma persiana em PVC, que ficou aberta durante a realização do estudo, e folhas de vidro, que ficaram fechadas, proporcionando assim um vão com camadas homogêneas. Para impedir a movimentação e a vibração da esquadria e, consequente, um ruído secundário que poderia interferir nos resultados, foi realizado um preenchimento entre as folhas. $\mathrm{O}$ fechamento vertical do vão da janela foi confeccionado com dois painéis em MDF, com $6 \mathrm{~mm}$ de espessura cada, e posicionado na face interna da abertura.

As amostras dos materiais ensaiados foram posicionadas, uma de cada vez, entre as duas placas de MDF, gerando assim uma divisória do tipo sanduiche, sendo que o material ensaiado o recheio. Para impedir que o ruído passasse pela folga entre a janela e a divisória, e melhorar a estanqueidade da câmara de recepção, foi colocada fita autoadesiva de PVC. A seguir, a Figura 01 apresenta a janela com o painel confeccionado para a realização dos testes aberto (A); as amostras de um dos materiais ensaiados, com o painel interno removido (B); e o fechamento composto já montado, pronto para a realização dos ensaios. A Figura 02 representa, esquematicamente, um corte da janela e do painel. 
Figura 01 - Vista do painel aberto (A); vista do painel aberto com o recheio de PU $10 \%$ (B); e vista do painel pronto para a realização do ensaio (C).

(A)

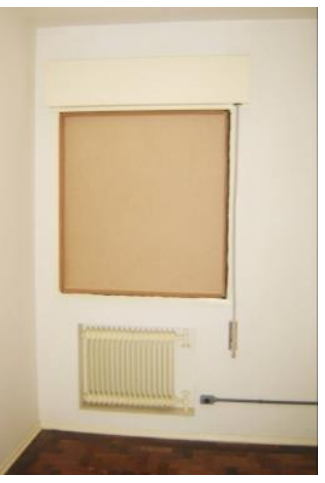

(B)

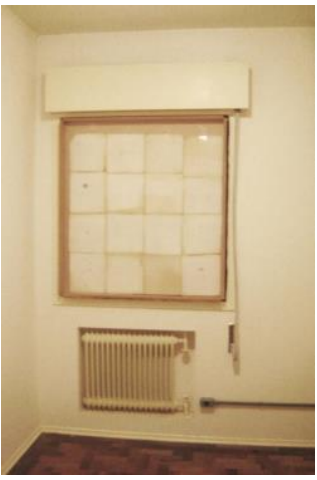

(C)

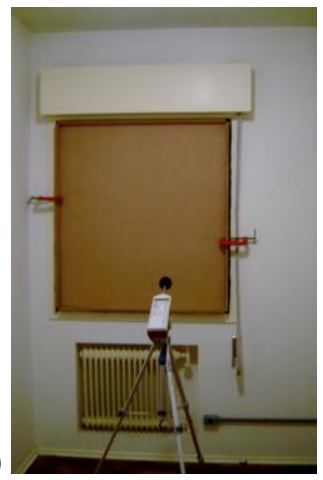

Figura 02 - Corte esquemático da janela e painel de vedação composto.
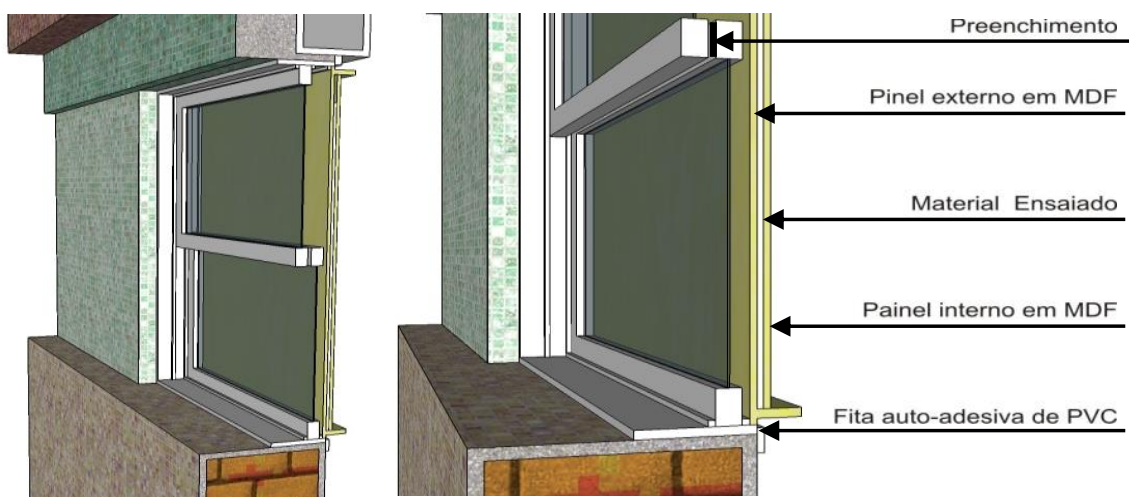

As medições foram realizadas de acordo com o método global da ISO 140-5 para elementos de vedações verticais externas e fachadas. Uma única alteração com relação a esta norma foi realizada: Ao invés de serem adotadas cinco ou mais posições para o analisador, optou-se por apenas três pontos devido ao tamanho reduzido da câmara de recepção. Esta alteração foi realizada, pois se as leituras dos dados fossem feitas em cinco pontos eles seriam muito próximos um do outro e não apresentariam diferenças consideráveis. Os equipamentos foram posicionados para a realização do ensaio conforme mostra a Figura 3, a seguir.

Figura 03 - Representação esquemática da posição dos equipamentos de medição

\section{em planta baixa e em corte.}

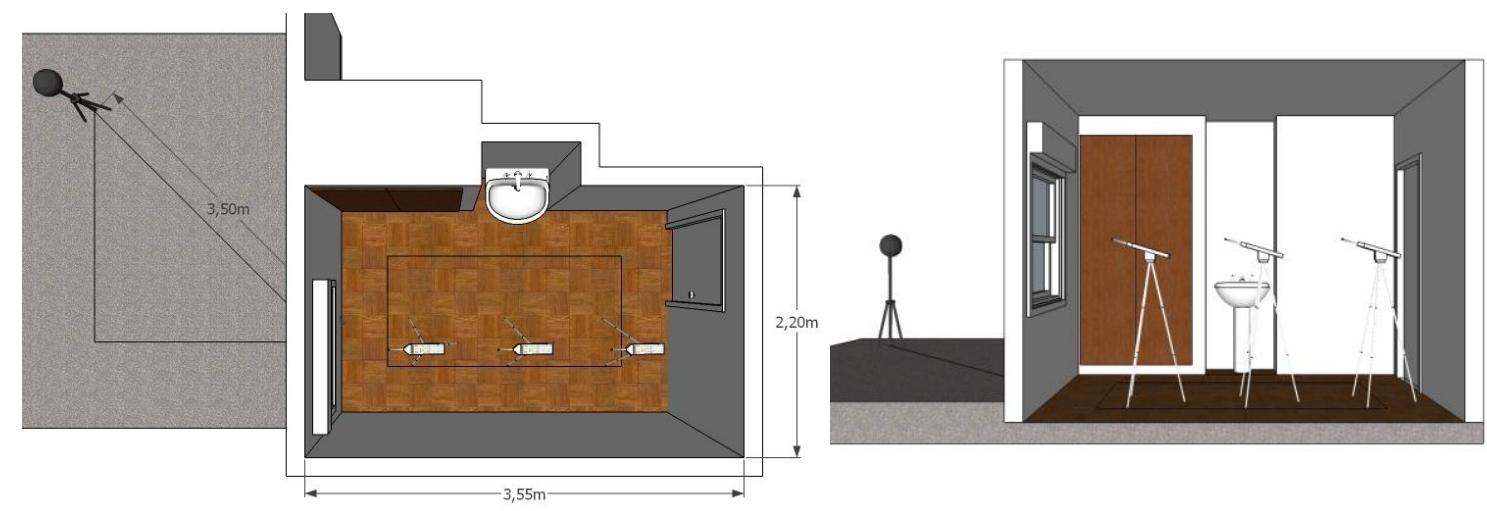


A fonte sonora omnidirecional foi posicionada a 3,5 $\mathrm{m}$ do centro geométrico da janela, com incidência de $45^{\circ}$ (Figura 43, 44 e 45). O analisador foi posicionado no interior da edificação, coletando-se os dados em três posições distintas do analisador com altura invariável de 1,5 m. Também foram respeitados os espaçamentos mínimos de 0,70 m entre posições de analisadores, 0,5 m entre posições do analisador e paredes do ambiente e 1,00 m entre qualquer posição do analisador e a fonte sonora. Os dados foram coletados três vezes por ponto. Após o posicionamento dos equipamentos foram, então, realizadas as medições do ruído de fundo. Como os valores encontrados não foram que foi superior a $10 \mathrm{~dB}$, não foi necessária correção, conforme a ISO 140-5. Posteriormente, já com a fonte sonora omnidirecional emitindo ruído rosa, se mediu o nível sonoro na câmara de recepção com analisador sonoro tipo 1. Os dados foram coletados três vezes em cada frequência em cada posição para cada configuração de fechamento, para que posteriormente fossem realizadas as avaliações.

Para a realização este estudo foi utilizado o "método de engenharia" descrito pela NBR 15575, que determina de forma rigorosa a isolação sonora global da vedação, caracterizando o comportamento acústico do sistema. Segundo essa norma, para determinar a diferença padronizada de nível ponderado (DnT,w) entre ambientes, devese utilizar o procedimento especificado na ISO 717-1, a partir dos valores de diferença padronizada de nível. Se os valores de DnT,w obtidos em ensaios estiverem de acordo com os valores apresentados da norma, a diferença padronizada de nível ponderada entre ambientes também estará de acordo com a norma.

\section{RESULTADOS}

O Quadro a seguir (1) apresenta um comparativo dos níveis de ruído obtidos nos ensaios para as diferentes configurações de vedações ensaiadas, descrevendo o nível $(\mathrm{dB})$ de cada frequência analisada. Os valores apresentados correspondem à média dos três valores obtidos nas medições para cada situação. A partir dos dados do Quadro foi gerado o gráfico da Figura 4, para facilitar a visualização dos resultados.

\section{Quadro 1 - Resultado das medições realizadas}

\begin{tabular}{|c|c|c|c|c|c|c|}
\hline $\mathbf{H z}$ & $\begin{array}{c}\text { Painel } \\
\text { Simples }\end{array}$ & $\begin{array}{c}\text { Lã de } \\
\text { Vidro }\end{array}$ & PU r 15\% & $\mathbf{3 ~ P u s}$ & $\begin{array}{c}\text { EVA r } \\
\mathbf{4 0 \%} \\
(\mathbf{2 0 x 1 8})\end{array}$ & $\begin{array}{c}\text { EVA r } \\
\mathbf{4 0 \%} \\
(\mathbf{9 0 x 7 0})\end{array}$ \\
\hline 100 & 50,5 & 49,4 & 48,7 & 49,2 & 48,9 & 49,3 \\
\hline 125 & 60,9 & 55,6 & 55,4 & 56,9 & 57,1 & 56,1 \\
\hline 160 & 61,1 & 57,8 & 58,2 & 58,8 & 58,1 & 58,3 \\
\hline 200 & 62,1 & 60,8 & 61,3 & 61,0 & 61,4 & 61,6 \\
\hline 250 & 60,8 & 57,1 & 58,4 & 58,9 & 58,1 & 59,3 \\
\hline 315 & 60,3 & 54,8 & 56,6 & 56,2 & 56,4 & 56,9 \\
\hline 400 & 54,9 & 48,5 & 52,3 & 50,3 & 51,7 & 55,7 \\
\hline 500 & 51,4 & 48,3 & 51,5 & 48,9 & 51,1 & 52,9 \\
\hline 630 & 48,1 & 44,0 & 47,9 & 44,2 & 47,1 & 48,7 \\
\hline 800 & 48,3 & 47,0 & 48,5 & 46,7 & 48,5 & 50,2 \\
\hline 1000 & 44,5 & 42,6 & 45,2 & 42,5 & 43,7 & 45,6 \\
\hline 1250 & 37,4 & 34,8 & 36,8 & 36,0 & 37,2 & 37,2 \\
\hline 1600 & 40,1 & 39,3 & 39,7 & 40,3 & 40,4 & 40,4 \\
\hline 2000 & 36,8 & 36,1 & 36,8 & 37,0 & 36,6 & 36,7 \\
\hline 2500 & 33,5 & 33,4 & 33,1 & 33,2 & 33,3 & 33,2 \\
\hline 3150 & 32,7 & 31,8 & 32,3 & 32,4 & 32,7 & 32,5 \\
\hline
\end{tabular}




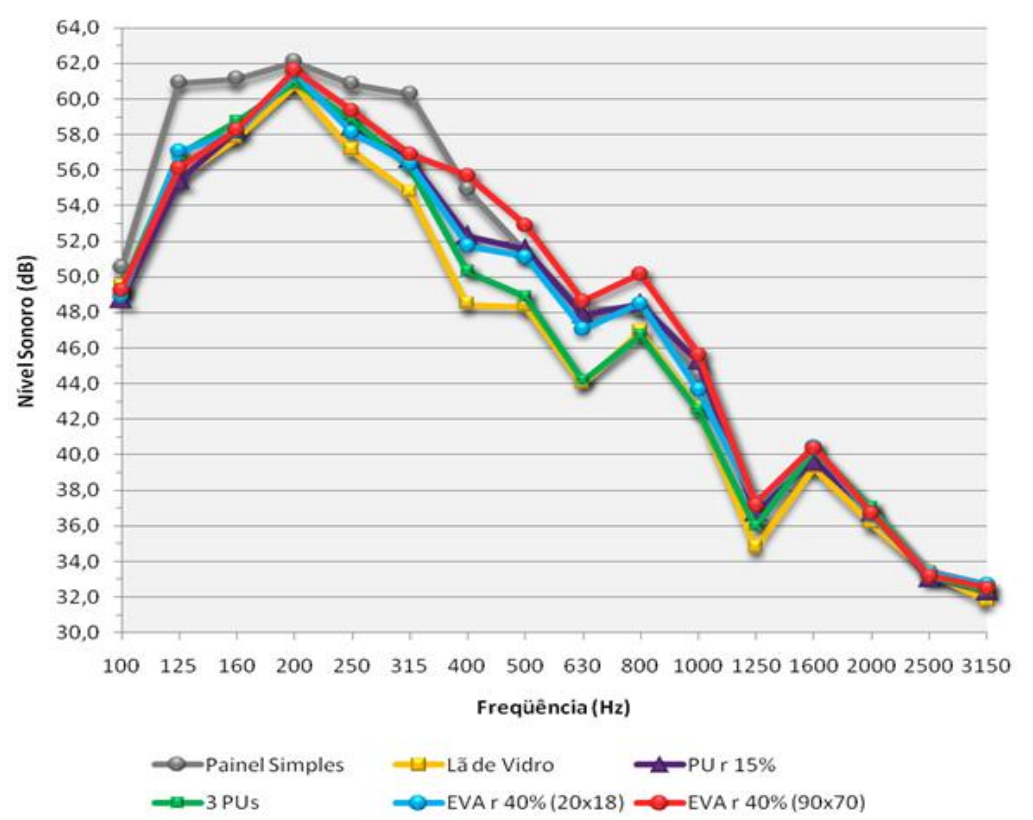

Ao analisar o Quadro 1 e o gráfico da Figura 4, pode-se observar que todas as configurações apresentaram comportamento semelhante nas frequências entre 100 e 200 $\mathrm{Hz}$, possuindo nível sonoro inferior ao painel simples, e, entre 1600 a $3150 \mathrm{~Hz}$ apresentam níveis aproximados ao do fechamento simples. Verifica-se que lã de vidro obteve o melhor desempenho, seguida pela divisória cujo recheio foi constituído por 3 PUs resíduos $(0 \%+5 \%+10 \%)$, que apresentou comportamento muito semelhante ao do material existente no mercado a partir de $630 \mathrm{~Hz}$. Observou-se também que o EVA resíduo $40 \%$ com placa de $90 \times 70 \mathrm{~cm}$ apresentou o pior desempenho entre os materiais isolantes ensaiados, ampliando o ruído em algumas frequências.

Outro fato que despertou atenção ao analisar o gráfico foi a crescente na frequência de $1600 \mathrm{~Hz}$, considerada frequência crítica (fc). A frequência crítica encontra-se neste ponto em função de o painel utilizado no ensaio ser de madeira com $0,6 \mathrm{~cm}$, coincidindo com o valor esperado. Isto explica o aumento do nível sonoro a $1600 \mathrm{~Hz}$ na câmara de recepção. Segundo Pujolle (1978) para evitar frequência crítica em divisórias compostas, os materiais utilizados devem ser diferentes ou possuírem espessuras diferentes, o que não acontece neste caso.

Seguindo o método adotado, foi calculado da diferença padroniza de nível (DnT), de acordo com a norma ISO 140-4. Para o cálculo foram utilizados os dados obtidos nas medições do ruído externo e calculado o tempo de reverberação. Os resultados são apresentados no Quadro 02.

\section{Quadro 2 - Resultado do DnT de cada material}

\begin{tabular}{|c|c|c|c|c|c|c|c|}
\hline $\begin{array}{c}\text { Ruído } \\
\text { Externo }\end{array}$ & TR & $\begin{array}{c}\text { DnT Painel } \\
\text { Simples }\end{array}$ & $\begin{array}{c}\text { DnT Lã de } \\
\text { Vidro }\end{array}$ & $\begin{array}{c}\text { DnT PU r } \\
\mathbf{1 5 \%}\end{array}$ & $\begin{array}{c}\text { DnT 3 } \\
\text { Pus }\end{array}$ & $\begin{array}{c}\text { DnT EVA r } \\
\mathbf{4 0 \%}(\mathbf{2 0 x 1 8})\end{array}$ & $\begin{array}{c}\text { DnT EVA r } \\
\text { 40\% (90x70) }\end{array}$ \\
\hline 71,067 & 1,45 & 25,157 & 26,257 & 26,946 & 26,457 & 26,746 & 26,435 \\
\hline 72,833 & 1,45 & 16,557 & 21,868 & 22,035 & 20,557 & 20,391 & 21,368 \\
\hline 76,333 & 1,5 & 19,982 & 23,327 & 22,871 & 22,349 & 22,960 & 22,838 \\
\hline 84,733 & 1,6 & 27,707 & 28,996 & 28,507 & 28,829 & 28,340 & 28,185 \\
\hline 84,467 & 1,66 & 28,856 & 32,578 & 31,278 & 30,811 & 31,600 & 30,345 \\
\hline
\end{tabular}




\begin{tabular}{|l|c|c|c|c|c|c|c|}
\hline 84,933 & 1,68 & 29,930 & 35,441 & 33,586 & 34,019 & 33,841 & 33,297 \\
\hline 81,367 & 1,5 & 31,216 & 37,671 & 33,871 & 35,827 & 34,405 & 30,427 \\
\hline 80,367 & 1,52 & 33,840 & 36,862 & 33,651 & 36,318 & 34,073 & 32,295 \\
\hline 76,700 & 1,62 & 33,683 & 37,783 & 33,928 & 37,605 & 34,739 & 33,150 \\
\hline 77,567 & 1,77 & 34,768 & 36,101 & 34,557 & 36,346 & 34,546 & 32,901 \\
\hline 71,833 & 1,87 & 33,029 & 34,940 & 32,318 & 35,040 & 33,895 & 31,918 \\
\hline 71,167 & 1,82 & 39,422 & 41,978 & 39,978 & 40,789 & 39,544 & 39,544 \\
\hline 71,767 & 1,73 & 37,035 & 37,869 & 37,502 & 36,835 & 36,713 & 36,746 \\
\hline 73,433 & 1,61 & 41,723 & 42,367 & 41,712 & 41,467 & 41,934 & 41,779 \\
\hline 75,400 & 1,6 & 46,985 & 47,085 & 47,329 & 47,240 & 47,107 & 47,251 \\
\hline 71,033 & 1,58 & 43,319 & 44,241 & 43,697 & 43,630 & 43,330 & 43,497 \\
\hline
\end{tabular}

Para avaliar o desempenho desses materiais de acordo com a NBR 15.575:2013 foi calculado o número único do isolamento ao ruído aéreo seguindo a ISO 717-1. Esse número é denominado diferença padronizada de nível ponderada (DnT,w). Após a obtenção do DnT,w de cada um dos materiais analisados, foi gerado um gráfico que permitiu uma avaliação comparativa dos desempenhos (Figura 5). O gráfico confirma que a lã de vidro obteve o melhor desempenho, pois apresentou DnT,w de $46 \mathrm{~dB}$, isolando, assim, 1,5 dB a mais que o fechamento simples. $\mathrm{O}$ segundo melhor desempenho foi dos 3 PUs resíduo $((0 \%+5 \%+10 \%)$, que isolou $0,5 \mathrm{~dB}$ a menos que o fechamento com lã de vidro. Em terceiro lugar empataram as amostras de PU resíduo $15 \%$ e de EVA resíduo $40 \%(20 \times 18 \mathrm{~cm})$, que isolaram $1 \mathrm{~dB}$ a menos. O EVA resíduo $40 \%(90 \times 70 \mathrm{~cm})$ apresentou o mesmo resultado que fechamento simples $44,5 \mathrm{~dB}$.

Figura 5 - Gráfico do DnT,w das configurações de fechamentos ensaiados.

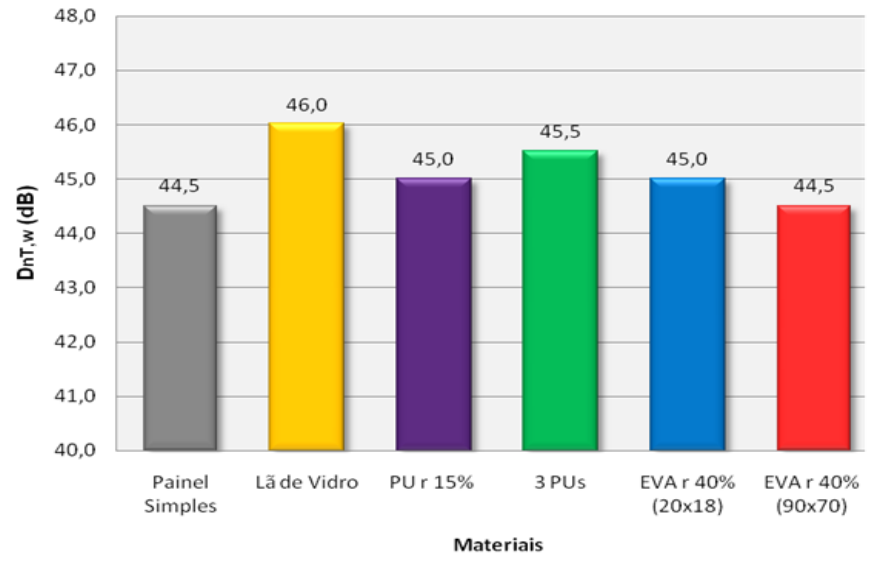

A partir dos dados encontrados foi possível, então, classificar o desempenho dos materiais ensaiados de acordo com a NBR 15575-4 (Quadro 3). Pode-se observar a partir do quadro que apenas um dos materiais alternativos testados no isolamento acústico fechamento composto, o EVA resíduo $40 \%$ de $90 \times 70 \mathrm{~cm}$, não atingiu o desempenho mínimo exigido na norma brasileira em duas situações. Não sendo adequado, portanto, o seu uso em paredes entre unidades habitacionais autônomas quando pelo menos um dos ambientes for dormitório, e em parede cega entre unidade habitacional e áreas comuns de permanência de pessoas, atividades de lazer e atividades esportivas. 


\section{Quadro 3 - Classificação dos materiais de acordo com a NBR 15575}

\begin{tabular}{|c|c|c|c|c|c|c|}
\hline \multirow[b]{2}{*}{ Elemento } & \multicolumn{6}{|c|}{ Nível de desempenho DnT,w (dB) } \\
\hline & $\begin{array}{l}\text { Painel } \\
\text { Simples } \\
44,5 \mathrm{~dB}\end{array}$ & $\begin{array}{l}\text { Lã de } \\
\text { Vidro } \\
\text { 46dB }\end{array}$ & $\begin{array}{l}\text { PU r } \\
15 \% \\
45 \mathrm{~dB}\end{array}$ & $\begin{array}{l}3 \text { Pus } \\
45,5 \\
\text { dB }\end{array}$ & $\begin{array}{l}\text { EVA r } \\
40 \% \\
(20 \times 18) \\
45 \mathrm{~dB}\end{array}$ & $\begin{array}{c}\text { EVA r } \\
40 \% \\
(90 \times 70) \\
44,5 \mathrm{~dB}\end{array}$ \\
\hline $\begin{array}{l}\text { Paredes entre unidades habitacionais } \\
\text { autônomas (paredes de geminação) nas } \\
\text { situações onde não haja ambiente dormitório }\end{array}$ & $\mathrm{M}$ & I & I & I & I & $\mathrm{M}$ \\
\hline $\begin{array}{l}\text { Paredes entre unidades habitacionais } \\
\text { autônomas (paredes de geminação) no caso de } \\
\text { pelo menos um dos ambientes ser dormitório }\end{array}$ & $\mathrm{N}$ & $\mathrm{M}$ & $\mathrm{M}$ & $\mathrm{M}$ & $\mathrm{M}$ & $\mathrm{N}$ \\
\hline $\begin{array}{c}\text { Parede cega de dormitórios entre uma unidade } \\
\text { habitacional e áreas comuns de trânsito } \\
\text { eventual, tais como corredores e escadaria nos } \\
\text { pavimentos }\end{array}$ & $\mathrm{M}$ & I & I & I & I & $\mathrm{M}$ \\
\hline $\begin{array}{c}\text { Parede cega de salas e cozinhas entre uma } \\
\text { unidade habitacional e áreas comuns de } \\
\text { trânsito eventual, tais como corredores e } \\
\text { escadarias nos pavimentos }\end{array}$ & $S$ & $S$ & $S$ & $S$ & $S$ & $S$ \\
\hline $\begin{array}{l}\text { Parede cega entre unidade habitacional e áreas } \\
\text { comuns de permanência de pessoas, atividades } \\
\text { de lazer e atividades esportivas, tais como } \\
\text { home theater, salas de ginástica, salão de } \\
\text { festas, salão de jogos, banheiros e vestiários } \\
\text { coletivos, cozinhas e lavanderias coletivas }\end{array}$ & $\mathrm{N}$ & $\mathrm{M}$ & $\mathrm{M}$ & $\mathrm{M}$ & $\mathrm{M}$ & $\mathrm{N}$ \\
\hline $\begin{array}{c}\text { Conjunto de paredes e portas de unidades } \\
\text { distintas separadas por um hall (DnT,w) obtida } \\
\text { entre as unidades }\end{array}$ & $\mathrm{M}$ & I & I & I & I & $\mathrm{M}$ \\
\hline
\end{tabular}

onde, $\mathrm{N}=$ não atingiu o mínimo;

$\mathrm{M}=$ mínimo;

$\mathrm{I}=$ intermediário;

$\mathrm{S}=$ superior.

O quadro 3 mostra que, exceto o EVA resíduo $40 \%$ de $90 \times 70 \mathrm{~cm}$, todos os materiais testados são adequados para o isolamento ao ruído aéreo em fechamentos composto, principalmente para as situações em que as exigências são tão elevadas, como Parede cega de dormitórios entre uma unidade habitacional e áreas comuns de trânsito eventual, tais como corredores e escadarias, por exemplo.

\section{CONSIDERAÇÕES FINAIS}

Analisando os resultados obtidos nos ensaios observa-se que é possível o uso de materiais alternativos e mais sustentáveis como estes, produzidos a partir de resíduos industriais. Apenas um dos materiais reciclados não atendeu a todos os requisitos da norma brasileira de desempenho, NBR 15575. Ainda assim, o uso deste material é adequado para situações em que as exigências não são tão elevadas.

Apesar de os materiais reciclados estudados não apresentarem um isolamento acústico tão elevado quanto o material convencional, a maioria dos materiais apresentou um bom desempenho. Este estudo demostra, portanto, o potencial do uso de materiais com polímeros reciclados para isolamento acústico em fechamentos compostos, e a 
necessidade de estimulo ao desenvolvimento e consumo de materiais mais sustentáveis como estes.

\section{REFERÊNCIAS}

ASSOCIAÇÃO BRASILEIRA DE NORMAS TÉCNICAS. Tratamento Acústico em Recintos Fechados. NBR 12179. Rio de Janeiro: ABNT, 2008.

ASSOCIAÇÃO BRASILEIRA DE NORMAS TÉCNICAS. Guia para Execução de Serviços de Medição de Ruído Aéreo e Avaliação dos Seus Efeitos Sobre o Homem. NBR 7731. Rio de Janeiro: ABNT, 1983.

NBR 15575: Edificações Habitacionais - Desempenho - Parte 4: Requisitos para os sistemas de vedações verticais internas e externas - SVVIE Apresentação. Rio de Janeiro: ABNT, 2013.

Acoustics - rating of sound insulation in buildings and of building elements. ISO 717. Gênova, Suíça: 1996.

BISTAFA, S. R. Acústica aplicada ao controle do ruído. São Paulo: Edgard Blucher, 2006. 368p.

CARVALHO, R. P. Acústica arquitetônica. Brasília: Theasaurus, 2006. 167p.

DIXIT, M. K.; FERNANDEZ-SOLIS, J. L.; CULP, C. H. Protocol for Embodied Energy Measurament Parameters. Departament of Architecture, Texas A\&M University, United States of America, 2010.

GARCIA, D. B.; VECCI, A. M.; RODRIGUES, F. C. Avaliação do isolamento sonoro aéreo de elementos de vedação na construção civil. Ambiente Construído, Porto Alegre, v. 8, n. 1, p. 49-63, jan./mar. 2008.

INTERNATIONAL ORGANIZATION FOR STANDARDIZATION. Acoustics Measurement of sound insulation in buildings and of building elements. ISO 140. Gênova, Suíça: 1998.

Menegotto, A. N. Avaliação de Materiais com Resíduos Poliméricos Para o Tratamento do Ruído De Impacto em Pisos. Caxias do Sul, 2009, 79p. Monografia (Curso de Arquitetura e Urbanismo) Universidade de Caxias do Sul, Caxias do Sul, 2010.

OITICICA, M. L. G. R.; BERTOLLI, S. R. Melhoria do desempenho acústico em peitoril ventilado. Acústica 2008. V. 1, p. 1-11, Coimbra, Portuga, 2008

PUJOLLE, J. La pratique de l'isolation acoustique des batiments. Paris: Moniteur, 1978.

RAUBER, J. Avaliação de Material com resíduos de EVA na Atenuação da Transmissão de Ruídos de Impacto em Pisos. Caxias do Sul, 2010,63p. Monografia (Curso de Arquitetura e Urbanismo) Universidade de Caxias do Sul, Caxias do Sul, 2010.

SILVA, D. C. Desempenho Acústico de Materiais à Base de Resíduos de PU para Redução de Ruídos de Impacto em Pisos. Caxias do Sul, 2010, 62p. Monografia (Curso de Arquitetura e Urbanismo) Universidade de Caxias do Sul, Caxias do Sul, 2010.

VALLE, S. Manual prático de acústica. 2.ed. Rio de Janeiro: Música \& Tecnologia, 2007. $373 p$. 